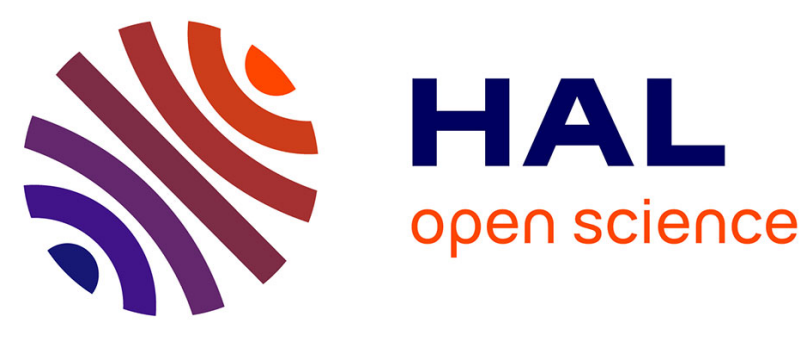

\title{
Template-directed synthesis of titania nanocages with four tetrahedrally arranged open windows
}

\author{
Jiaji Cheng, Nicolas Mallet, Walid Baaziz, Ovidiu Ersen, Emilie Gombart, \\ Valérie Alard, Jérôme Majimel, Marie-Hélène Delville, Mona \\ Tréguer-Delapierre, Etienne Duguet
}

\section{To cite this version:}

Jiaji Cheng, Nicolas Mallet, Walid Baaziz, Ovidiu Ersen, Emilie Gombart, et al.. Template-directed synthesis of titania nanocages with four tetrahedrally arranged open windows. Chemistry - A European Journal, 2018, 24 (27), pp.6917-6921. 10.1002/chem.201800186 . hal-01780700

\author{
HAL Id: hal-01780700 \\ https://hal.science/hal-01780700
}

Submitted on 15 May 2018

HAL is a multi-disciplinary open access archive for the deposit and dissemination of scientific research documents, whether they are published or not. The documents may come from teaching and research institutions in France or abroad, or from public or private research centers.
L'archive ouverte pluridisciplinaire HAL, est destinée au dépôt et à la diffusion de documents scientifiques de niveau recherche, publiés ou non, émanant des établissements d'enseignement et de recherche français ou étrangers, des laboratoires publics ou privés. 


\title{
Template-Directed Synthesis of Titania Nanocages with Four Tetrahedrally-Arranged Open Windows
}

\author{
Dr. Jiaji Cheng, ${ }^{[a]}$ Nicolas Mallet, ${ }^{[a]}$ Dr. Walid Baaziz, ${ }^{[b]}$ Prof. Ovidiu Ersen, ${ }^{[b]}$ Dr. Emilie Gombart, ${ }^{[c]}$ Dr. Valérie Alard, ${ }^{[c]}$ \\ Dr. Jérôme Majimel, ${ }^{[a]}$ Dr. Marie-Hélène Delville, ${ }^{*[a]}$ Prof. Mona Tréguer-Delapierre, ${ }^{*[a]}$ and Prof. Etienne Duguet ${ }^{*[a]}$ \\ [a] CNRS, Univ. Bordeaux, ICMCB, UMR 5026, 33600Pessac (France) / ${ }^{[b]}$ Institut de Physique et Chimie des Matériaux de Strasbourg (IPCMS) \\ UMR 7504 CNRS, Université de Strasbourg, 23 rue du Loess, F-67034, Strasbourg Cedex 2 (France) / ${ }^{[c]}$ Département Innovation Matériaux, \\ LVMH Recherche, 45804 Saint Jean de Braye (France).
}

\begin{abstract}
Original titania nanocages are fabricated from sacrificial silica/polystyrene tetrapod-like templates. Here the template synthesis, titania deposition and nanocage development through polystyrene dissolution and subsequent silica etching are described. Discussion about the competitive deposition of titania on the biphasic templates is particularly emphasized. The morphology of the nanocages is investigated by TEM, STEM, EDX mapping and electron tomography.
\end{abstract}

Nanoparticles / Electron tomography / Nanocage / Sol-gel chemistry / Template-based synthesis / Titania

The rapid development of nanoscience is supported by the design and fabrication of nanoparticles, which are becoming more and more complex so as to particularly tackle multifunctionality issues. ${ }^{[1]}$ Among these designs, hollow nanoparticles are being intensively studied as a new generation of components for device fabrication, due to the high surface area, multiple scattering properties, shell permeability and large light-harvesting efficiency when compared with their solid counterparts. ${ }^{[2]}$ For instance, it was reported that through hydrothermal reaction, a uniform titania $\left(\mathrm{TiO}_{2}\right)$ shell could be deposited on $\mathrm{Cu}_{2} \mathrm{O}$ polyhedra to form discrete $\mathrm{Cu}_{2} \mathrm{O} @ \mathrm{TiO}_{2}$ core/shell structures with controlled size and uniform shell. ${ }^{[3]}$ The hollow morphology was then developed by acidic etching of the $\mathrm{Cu}_{2} \mathrm{O}$ template. One decade ago, nanocages appeared which are defined here as hollow nanoparticles with open windows. The most famous ones are gold nanocages, obtained in one step by a galvanic replacement reaction when sacrificial silver nanocubes react with $\mathrm{HAuCl}_{4}{ }^{[4]}$ Regular nanocages were obtained by the simultaneous or sequential action of galvanic replacement and the Kirkendall effect. ${ }^{[5]}$ However, the as-synthesized nanocages are exclusively metallic. Moreover, the precise control of the nanostructures in terms of the number, position and dimensions of the windows, as well as the versatility of the protocol so as to obtain inorganic nanocages of any chemical composition with high morphological yield still remain challenging.

To tackle these restrictions, we extended the initial strategy of surface coating on, and subsequent etching of, sacrificial templates, by envisioning templates that are more complex. The latter would be made 
of two phases with different surface properties. One phase shows affinity for the coating material, contrary to the other, in order to achieve a discontinuous shell and therefore simultaneously carve the hollow core and the open windows. Indeed, through fine control of a seeded-growth emulsion polymerization reaction, highyield batch syntheses of binary tetrapods, hexapods and dodecapods, with silica cores and polystyrene (PS) satellites have been achieved. ${ }^{[6]}$ Such binary clusters are convenient templates for achieving new heterogeneous nanomaterials. For example, we showed that it is possible to deposit a homogenous and continuous silica coating on the whole silica/PS clusters by pre-anchoring silanol groups on the PS satellites. ${ }^{[7]}$ Moreover, the pristine PS satellites being unreactive towards the silica precursors, we demonstrated that the silica deposit could be regioselectively restricted to the accessible surface of the silica core ${ }^{[8]}$ Therefore, dissolving the PS nodules in tetrahydrofuran (THF) revealed silica particles displaying surface dimples, that is, indentations, where the number and position directly depend on those of the PS satellites, and with a depth correlated to the deposited silica thickness.

Herein, we present a feasible and versatile strategy for the synthesis of metal oxide nanocages, with tuneable size and number of windows, based on these well-established PS/silica multipod-like templates. ${ }^{[6 \mathrm{~b}]}$ In brief, we investigated the fabrication of titania hollow nanocages with four open windows by templateassisted deposition of titania shells on PS/silica tetrapods. The full synthetic strategy consists of three main steps: template fabrication, titania deposition and nanocage development (Figure1).

A $5 \mathrm{~g}$ batch of PS/silica multipods was fabricated from $55 \mathrm{~nm}$ silica seeds according to an optimized protocol. ${ }^{[6]}$ The batch was essentially made of tetrapods, that is, $84 \%$ with regard to the silica seeds (Figure 2). Their geometrical features were $55 \mathrm{~nm}$ for the silica core diameter and $145 \mathrm{~nm}$ for the average diameter of the PS satellites. It is worth mentioning that these four satellites are tetrahedrally-arranged because their positions were optimized by their steric repulsion during their growth on the limited surface area of the seed.

Prior to titania deposition around the PS/silica templates, the surface of the silica core, previously modified with methacryloxymethyltrimethoxysilane to promote the nucleation and growth of the PS satellites, was made organic-free again by depositing a new layer of silica. This regrowth process was performed by the slow addition and in situ polycondensation of tetraethoxysilane (TEOS) in the presence of ammonium hydroxide in a water/ethanol mixture. ${ }^{[8]}$ The amount of TEOS was adjusted to create a silica layer of $10-12 \mathrm{~nm}$, bringing the diameter of the silica cores to $80 \mathrm{~nm}$. It may be emphasized that TEOS polycondensation occurred only on the silica core, while the average diameter of the PS satellites remained unchanged. It confirms that regioselective surface modification of such biphasic templates is feasible. 
According to the same strategy, we attempted the regioselective deposition of a second coating made of titania. It was expected to be more difficult, because the reactivity of titanium alkoxides is much higher than that of silicon alkoxides, for example, TEOS. Actually, for silicon alkoxides hydrolysis-condensation rates must be increased by catalysts, for example, ammonia, while the same reactions for titanium alkoxides must be slowed down with chemical additives, called retardants. Acetylacetone (Acac) is a complexing ligand well known to make the Ti--Acac bond less hydrolysable. Hence, the reactivity of titanium alkoxides is generally controlled through the molar hydrolysis ratio $h=\mathrm{H}_{2} \mathrm{O} / \mathrm{Ti}$ and complexation ratio $x=\mathrm{Acac} / \mathrm{Ti}$. Moreover, it was expected in the present situation of template-mediated process that the titania precursor-totemplate ratio would also be a critical parameter, especially for controlling the thickness of the titania shell. In brief, it was mandatory to slow down the polycondensation rate, not only to avoid homogeneous nucleation and precipitation, but also to promote a selective coating onto the silica cores. Titanium tetraisopropoxide (TTIP) was chosen as titania precursor. Preliminary experiments gave information on the influence of the parameters $x, h$ and the ratio of tetrapod-like template to TTIP, and demonstrated that they were more or less interdependent. First, the use of Acac is mandatory to promote template-mediated precipitation, that is, to avoid homogeneous precipitation of titania in solution. Second, the homogeneous precipitation prevails also at high $h$ value (Figure 3 ). When $h=200$, many free titania nanoparticles were generated and the thickness of the titania coating onto the template remained low. Thirdly, lower $h$ value and/or to some extent higher $x$ value increased PS satellite coalescence and decreased the thickness of the titania coating (Figure 3 for $h=0$ ). This means that there was an obvious competition between the TTIP hydrolysis and its capture by the PS nodules, which were hence swollen, and in the end the ultimate loci for the titania precipitation. This phenomenon is enhanced at low $h$ value, because TTIP and its complex with Acac are organophilic by nature and therefore good plasticizers for PS. As a consequence, the optimized parameters of the protocol to simultaneously prevent the homogeneous precipitation of titania and PS nodule coalescence, and to create a homogeneous $10 \mathrm{~nm}$--but unfortunately non regioselective--titania coating, are $x=2, h=100$ and a volume of $100 \mu \mathrm{L}$ of TTIP for $2.4 \times 10^{12}$ tetrapod-like templates.

The development of the titania nanocages was carried out in two steps. The first step consisted of the dissolution of the PS nodules. The use of good solvents for PS, for example, dimethylformamide (DMF) or THF, was expected to simultaneously remove the physically entangled macromolecules of the PS satellites and fragment their titania coating thanks to the internal pressure induced by the partial swelling of the nodules with solvent molecules. The applied protocol successively used DMF and THF and was shown to 
efficiently remove the PS nodules. However, it became less easy to completely fragment the titania coating thicker than $6 \mathrm{~nm}$, as already achieved (Figure 3). ${ }^{[8]}$ In such situations, we successfully applied a high-power ultrasonic treatment ( $150 \mathrm{~W}$ for $4 \mathrm{~h}$ ) to complete the fragmentation and hence refine the shape of the silicafilled titania nanocages (See Supporting Information Figure S2).

Finally, titania nanocages were achieved by further chemical etching the silica cores. It was mandatory to implement a selective etching process to preserve the titania component. After unsuccessful attempts with hydrofluoric acid (even at concentration as low as 2 wt. $\%$ and short contact times), we chose alkaline conditions with sodium hydroxide at $\mathrm{pH} \approx 12$, inspired by a protocol initially reported to fabricate hollow silica nanoparticles. ${ }^{[9]}$ To look deeper into the kinetics and selective efficacy of the chemical etching process, the morphology evolution with time was followed by TEM. This showed first that the silica cores were actually being attacked little by little, as it was observed that the diameter of the silica spheres progressively decreased (Figure 4). More surprisingly, it appeared that, even if they kept their spherical shape, the size-decreasing silica cores were systematically present within the titania nanocage, even when their diameter was lower than the diameter of the windows through which they might escape. Finally, a treatment of at least $24 \mathrm{~h}$ was necessary to bring the diameter to a negligible value. Second, the overall shape and size of the particles were kept unspoiled, meaning that the etching action is limited to the silica core while preserving the titania skeleton. This led to self-standing titania nanocages with an overall diameter of about $100 \mathrm{~nm}$.

The distribution of titania and silica within the nanocages was further investigated by energydispersive X-ray spectroscopy (EDX), coupled with scanning transmission electron microscopy (STEM) to map the $\mathrm{Ti}, \mathrm{Si}$ and $\mathrm{O}$ elements of a nanocage before and after $24 \mathrm{~h}$ etching (Figure 5). The Ti map and profile through the nanoparticle are fully consistent with the expected four-window nanocage morphology in both cases. Before etching, Si element was essentially situated at the centre of the titanium-based nanocage. Nevertheless, after $24 \mathrm{~h}$ etching, $\mathrm{Si}$ is still present and the Si profile and map are quite similar--but less intense--than that of Ti. This means that this nanocage is hollowed, but silicate species have probably precipitated again in a quite regular way onto the titania skeleton of the nanocage. This may explain why the Si:Ti ratio did not fall below $15 \%$ after $24 \mathrm{~h}$ etching, even if the silica cores were no longer observed on the TEM images. Likewise, electron tomography (ET) experiments were carried out on the titania nanocages which allows to observe in more detail their internal structure (Figure 6). The ET analysis performed on a typical nanocage highlights the presence of a silica residue located on both sides (internally and externally) 
of the titania structure. Traces of silica were clearly observed on the orthogonal slices extracted from the reconstructed volume (Figure $6 \mathrm{c}$ ) and evidenced on the 3D model (Figure $6 \mathrm{~d}$ ). These results are consistent with the above assumption of a silica solution/reprecipitation process described before. It should be emphasized that the as-obtained titania nanocages are particularly mechanically robust, despite the thickness of their walls (estimated to $10 \pm 5 \mathrm{~nm}$ ) and their amorphous structure, typically achieved in such low temperature processes. They remained structurally sound during centrifugation/redispersion cycles. This may be readily explained by their high degree of symmetry with four windows of similar size and circular shape arranged in a tetrahedral way creating an isotropic arching system. Extensive statistical analyses from TEM images were difficult to perform because of a lack of contrast between the large-field TEM images of the titania nanocages. Nevertheless, it is likely expected that nanocages with two or three windows co-exist with those of four windows because of the presence of low amounts of PS/silica bipods and tripods in the template batch.

In conclusion, we report a novel template synthesis approach to prepare titania nanocages never previously achieved. Our initial elegant strategy of titania regioselective deposition was revealed to be more difficult than expected, because of 1) the great difference of reactivity between silicon and titanium alkoxides, and 2) part of the template surface was of polymeric nature. Nevertheless, an alternative route to the targeted shape was achieved thanks to ultrasonic treatments. The as-obtained titania nanocages are actually hollow, equipped with four open windows in a tetrahedral arrangement, and mechanically robust, for example, supporting centrifugation cycles. This allows potential applications to be envisioned such as photocatalysts, because the self-standing morphology of the nanocages and the presence of the open windows should make easier the chemical circulation, and maximize the contact area with the active surface. Moreover, this original synthesis strategy using multipod-like biphasic templates might be easily extended to nanocages, not only with a different number of windows, but also with other chemical compositions.

\section{Acknowledgements}

This work was partially supported by LVMH Recherche company. A. Désert, P. E. Rouet and H. Palis are gratefully acknowledged for providing the silica/polystyrene templates, as well as A. Kabbara and M. Malivert for checking the repeatability of the synthesis procedures. All the TEM and STEM EDX measurements were performed at the Plateforme de Caractérisation des Matériaux (UMS 3626, Pessac, France).

\section{Conflict of interest}

The authors declare no conflict of interest. 


\section{Bibliography}

[1] a) Y. Wang, Y. Wang, X. Zheng, G.-R. Yi, S. Sacanna, D. J. Pine, M. Weck, J. Am. Chem. Soc. 2014, 136, 6866 -6869; b) D. Chen, R. A. Caruso, Adv.

Funct. Mater. 2013, 23, 1356- 1374; c) Y. Boyjoo, M. Wang, V. K. Pareek, J. Liu, M. Jaroniec, Chem. Soc. Rev. 2016, 45, 6013 6047.

[2] a) F. Caruso, R. A. Caruso, H. Mçhwald, Science 1998, 282, 1111 -1114; b) J. Hu, M. Chen, X. Fang, L. Wu, Chem. Soc. Rev. 2011, 40, 5472 - 5491; c) P. Zhang, A. Li, J. Gong, Particuology 2015, 22, 13-23; d) J. Liu, S. Z. Qiao, J. S. Chen, X. W. Lou, X. R. Xing, G. Q. Lu, Chem. Commun. 2011, 47, $12578-12591$.

[3] a) Z. Wang, X. W. D. Lou, Adv. Mater. 2012, 24, 4124 -4129; b) G. Jiang, X. Wang, Y. Zhou, R. Wang, R. Hu, X. Xi, W. Chen, Mater. Lett. 2012, 89, 59-62.

[4] S. E. Skrabalak, J. Chen, Y. Sun, X. Lu, L. Au, C. Cobley, Y. Xia, Acc. Chem.Res. 2008, 41, 1587 -1595.

[5] E. Gonz_lez, J. Arbiol, V. F. Puntes, Science 2011, 334, 1377- 1380.

[6] a) A. Perro, E. Duguet, O. Lambert, J. C. Taveau, E. Bourgeat-Lami, S. Ravaine, Angew. Chem. Int. Ed. 2009, 48, 361- 365; Angew. Chem. 2009, 121,

367-371; b) A. D_sert, J. Morele, J. C. Taveau, O. Lambert, M. Lansalot, E. Bourgeat-Lami, A. Thill, O. Spalla, L. Belloni, S. Ravaine, E. Duguet, Nanoscale

$2016,8,5454-5469$.

[7] A. D_sert, C. Hubert, A. Thill, O. Spalla, J.-C. Taveau, O. Lambert, M. Lansalot, E. Bourgeat-Lami, E. Duguet, S. Ravaine, Mol. Cryst. Liq. Cryst. 2014,

604, 27-32.

[8] A. D_sert, C. Hubert, Z. Fu, L. Moulet, J. Majimel, P. Barboteau, A. Thill, M. Lansalot, E. Bourgeat-Lami, E. Duguet, S. Ravaine, Angew. Chem. Int. Ed.

2013, 52, 11068 -11072; Angew. Chem. 2013, 125, $11274-11278$.

[9] Q. Zhang, T. Zhang, J. Ge, Y. Yin, Nano Lett. 2008, 8, 2867- 2871. 


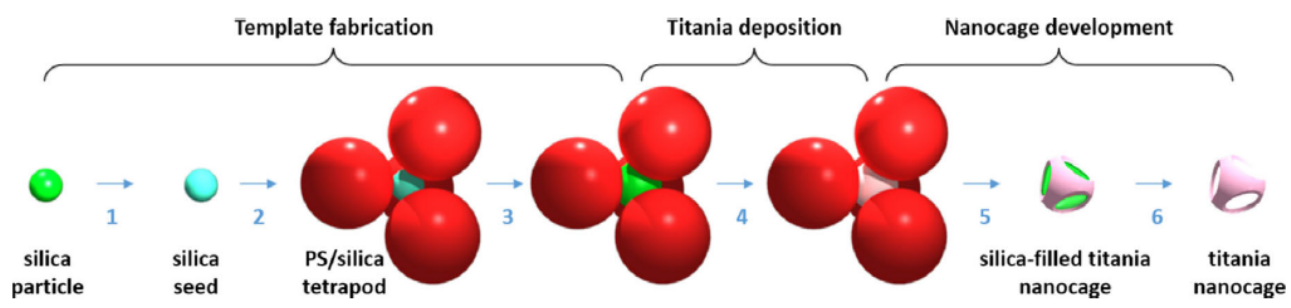

Figure 1. Considered synthetic route to titania nanocages with four open windows. Template fabrication steps include: 1) seed preparation by surface functionalization of $55 \mathrm{~nm}$ silica particles with methacryloxymethyltrimethoxysilane, 2) seed-growth emulsion polymerization of styrene and 3) regioselective regrowth of silica cores. The titania deposition step consists of 4) regioselective condensation of titania precursor. The nanocage development step includes 5) dissolution of PS nodules with THF and 6) silica core etching with alkaline solution.

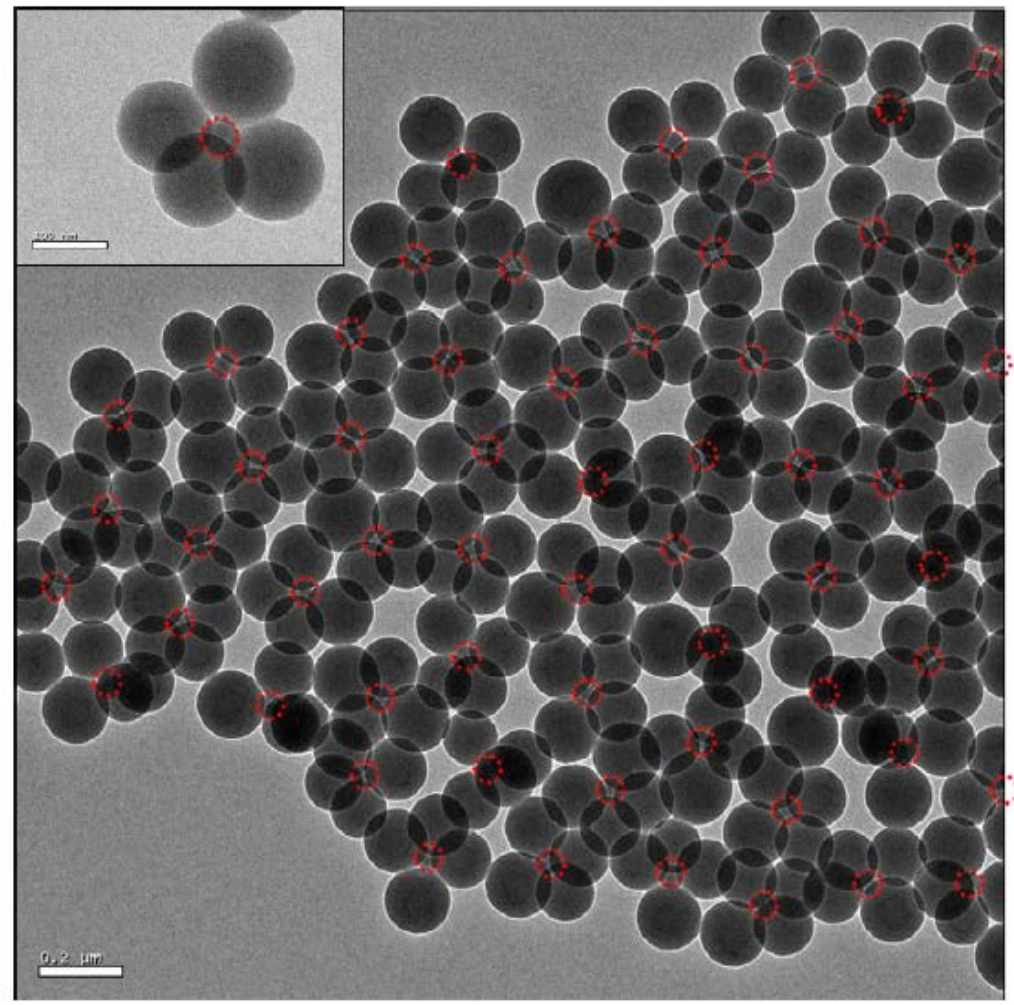

Figure 2. TEM image of the PS/silica tetrapod-like templates. Scale bar: $200 \mathrm{~nm}$ and $100 \mathrm{~nm}$ for inset. The silica cores are delineated by a red dotted circle for the sake of clarity (original TEM image in Supporting Information Figure S1). The main side-products (not observable on the TEM image) are tripods ( $11 \%$ ), bipods ( $2 \%$ ) and multi-silica clusters $(3 \%)$ as checked by extensive statistical analysis from TEM images. ${ }^{[6]]}$. 


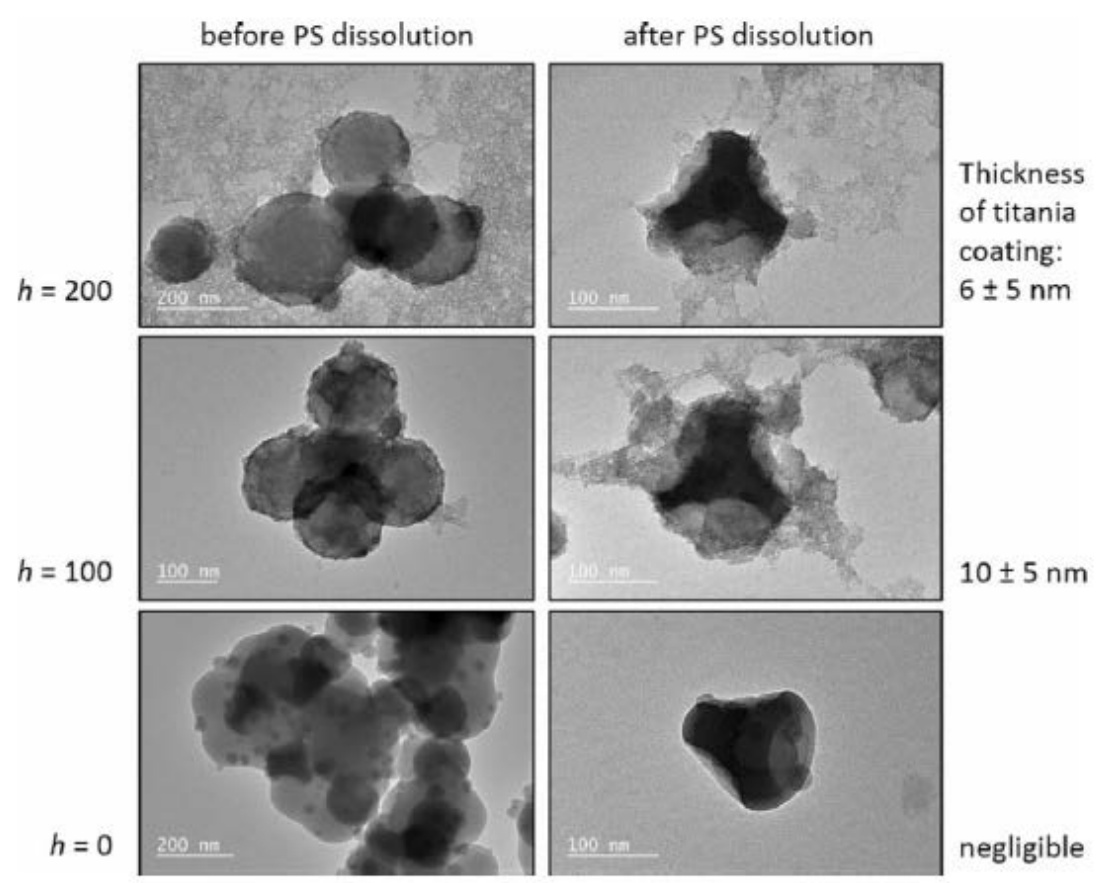

Figure 3. TEM images showing how the molar hydrolysis ratio $h=\mathrm{H}_{2} \mathrm{O} / \mathrm{TTIP}$ influences the titania coating morphology on the PS/silica tetrapod-like templates before ( $1^{\text {st }}$ row) and after dissolution of the PS satellites ( $2^{\text {nd }}$ row $)$. Experimental conditions: $2.4 \times 10^{12}$ tetrapod-like templates; $100 \mu \mathrm{L}$ TTIP; molar complexation ratio $x=A c a c / T T I P=2$. The titania thickness was estimated from measurements performed on the edges of more than 50 nanoparticles.
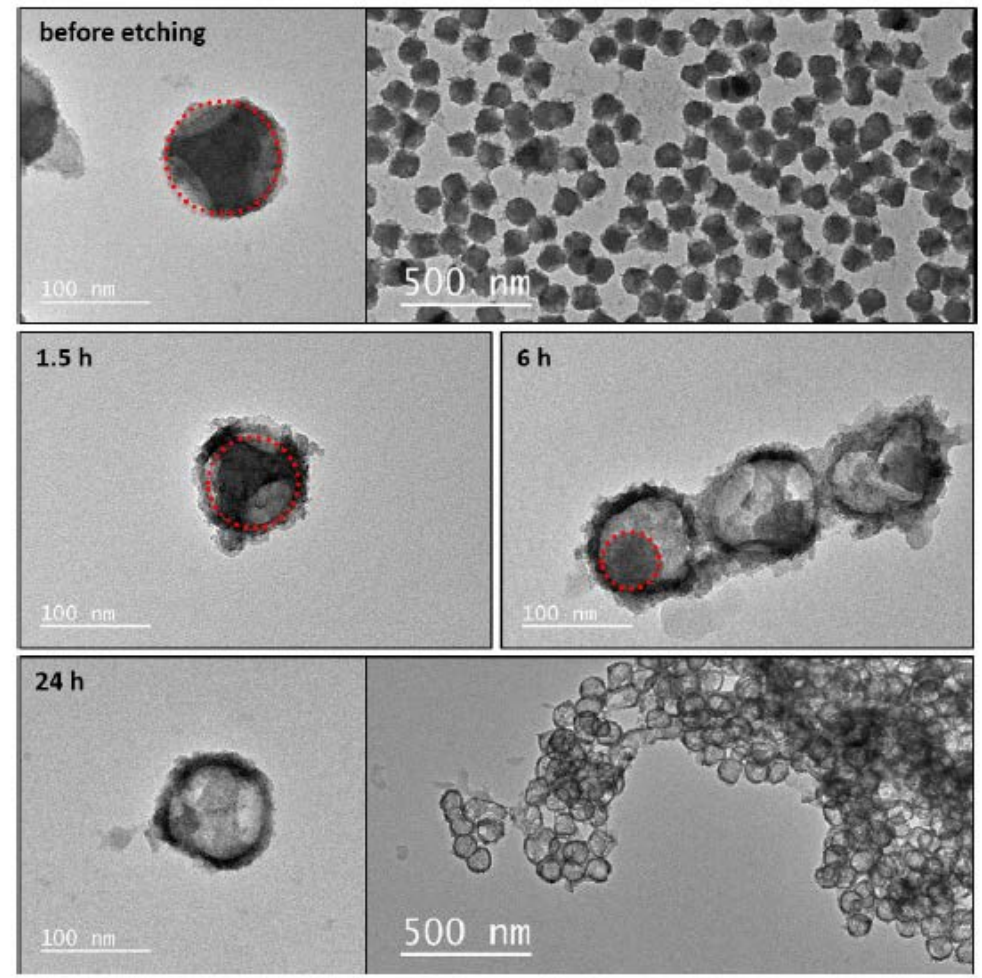

Figure 4. TEM images showing how the silica core (delineated by a red dotted line on images at high magnification) is progressively etched by $\mathrm{NaOH}$ with time, to provide titania nanocages. 
a)
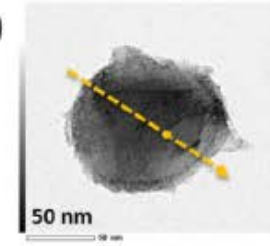

b)

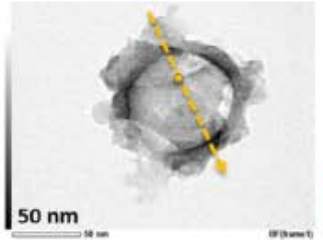

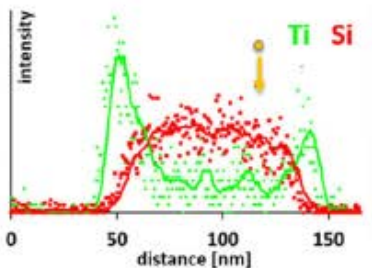

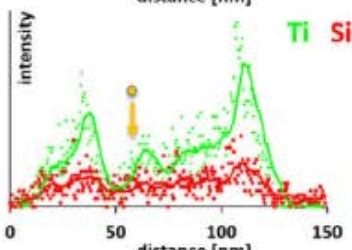

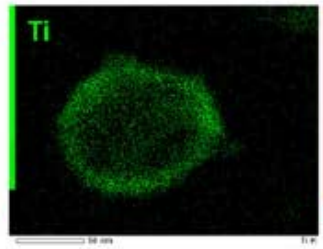

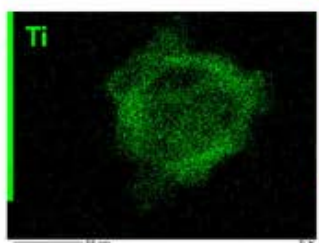

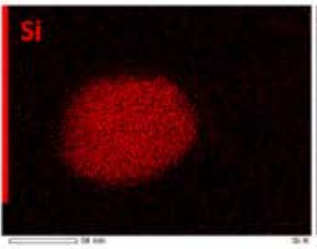

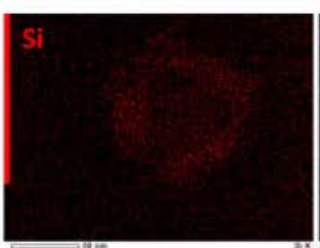

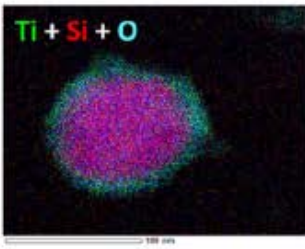

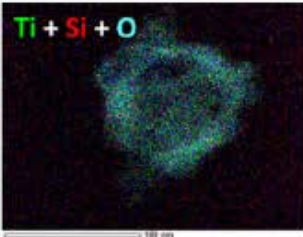

Figure 5. STEM images and EDX analyses of titania nanocages a) before and b) after $24 \mathrm{~h}$ etching of the silica core. 1 st row: STEM images; 2 nd row: plot of the titanium and silicon profiles along the dotted orange line drawn on STEM image; 3rd to 5th rows: STEM EDX mapping of titanium, STEM EDX mapping of silicon and overlay of titanium, silicon and oxygen STEM EDX maps, respectively.
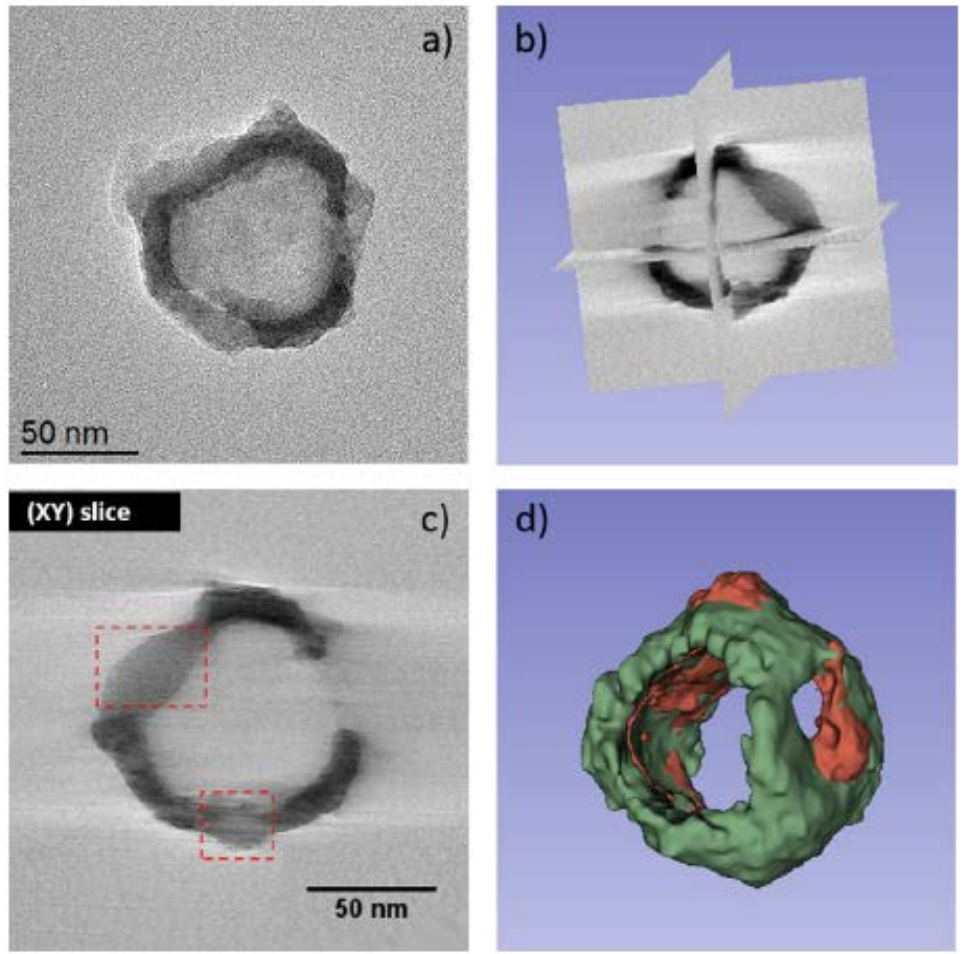

Figure 6. Electron tomography analysis of a titania nanocage: a) typical TEM image from the tilt series acquired on a titania nanocage chosen for the 3D analysis, b) orthogonal slices through the reconstructed volume of the nanostructure, c) $(X Y)$ slice from the reconstructed volume evidencing the presence of silica residue in the parts highlighted by red lines, d) 3D model of the corresponding titania nanocage showing its biphasic nature (titania in green and silica in red). 